\title{
The Palomar-Leiden Survey of Faint Minor Planets: Conclusion
}

\author{
C. J. VAN HOUTEN
}

Leiden Observatory, Wassenaarseweg 78, 2300 RA Leiden, The Netherlands

\author{
P. HERGET ${ }^{\dagger}$
}

AND

\section{B. G. MARSDEN}

Harvard-Smithsonian Center for Astrophysics, Cambridge, Massachusetts 02138

Received November 10, 1983; revised March 26, 1984

\begin{abstract}
The results are given of a revision and small extension of the Palomar-Leiden Survey of minor planets. The majority of the class 4 orbits in the original Survey have been rederived using positions measured from the second plate of each blink pair, and in some cases the orbits could be raised to class 1 quality by the identification of observations in the second month. By using the concept of " $e$-assumed orbits," meaningful-if not always accurate-orbits are given for the cases that previously had to be rejected. The extension to the Survey consists of 170 new objects found in the field used for photometric calibration purposes. The total number of orbits in the Survey is 2403, and a tabulation is given of the 1198 orbits that supplement or amend those in the original Survey. A listing is included of the identifications of Palomar-Leiden objects with minor planets observed at other oppositions.
\end{abstract}

\section{INTRODUCTION}

The Palomar-Leiden Survey of faint minor planets, published almost a decade and a half ago (van Houten et al., 1970; hereinafter called PLS I), had as its principal objective a statistical discussion of the orbits of faint minor planets. The orbits were ascribed to four quality classes depending on the available observations. Class 1 orbits were based on at least two positions in each observing month (September and October 1960). Class 2 orbits involved only a single position in one or the other of the months. Class 3 and 4 orbits were obtained from positions in one month only and were defined according as to whether the arc of observation was greater than or less than 7 days. PLS I consisted of 1965 orbits, including 129 entries that were rejected for reasons specified there.

\footnotetext{
$\uparrow$ Deceased; formerly the director of the Cincinnati Observatory.
}

During the first Tucson colloquium on minor planets in 1971 considerable interest was shown in the individual orbits in the Survey. For that reason an attempt has been made to improve the quality of the orbits, mainly by examining the Survey plates for further positions. It has been possible to transfer to an improved quality class some 120 of the orbits in PLS I, and for the majority of the orbits remaining in class 4 the opportunity was taken of using positions measured on the second plate of a blink pair. No systematic search for additional minor planets has been made in the original Survey fields, but there is a considerable increase in the number of fourthclass orbits on account of the acquisition of additional positions (from the September 1960 plates) of objects for which there had previously not been enough observations to permit an orbit determination. Furthermore, the plates containing Selected Area (SA) 68, which had been used for the photometric calibration, were also searched for 
minor planets. The minor planets on the SA 68 plates and the images of PLS I objects on additional nights were found in Leiden using a blink microscope lent by the Lunar and Planetary Laboratory. The number of orbits has in fact been increased by 441 , including 170 (designated with P-L numbers in the $3000 \mathrm{~s}$ ) determinations for objects found in the SA 68 field.

The total number of orbits in PLS II is 2403, and almost exactly half of the orbits represent new computations. More than 700 of the new orbits were computed by the late P. Herget. After Herget's death, B. G. Marsden obtained orbits for some 450 more objects. A number of orbits were derived at the Leiden Observatory by means of a computer program written by Herget, and a few orbits were computed by C. M. Bardwell, then at the Cincinnati Observatory.

\section{$e$-ASSUMED ORBITS}

In view of the low accuracy of the fourthclass orbits (and to some extent third-class orbits), two selection rules were applied for their acceptance in PLS I. These were that the orbital eccentricity $e$ should not exceed 0.30 and that the semimajor axis $a$ should not be smaller than 2.0 AU, except for objects of Hungaria type and the obvious Apollo-type object 6743 P-L. Similar rules have been adopted in PLS II, but the limits were changed slightly. The upper limit for $e$ was generally increased to 0.35 , since about $1 \%$ of the class 1 orbits have $e>0.30$. The general lower limit of $a$ was shifted to a more realistic $2.1 \mathrm{AU}$, and objects that seemed to be at and beyond the 2:1 Kirkwood gap (3.3 AU) were subjected to careful scrutiny. The relaxation of the eccentricity limit has meant that five of the previously rejected class 3 orbits can be accepted without change. These are 7620 , 9086, 9528, 9552, and 9593 P-L: the reliability of the second of these orbits has in fact been demonstrated by the identification of observations of the same object in 1980.

The total number of orbits rejected according to the new limits was 91, while for 13 further objects no orbit could be deter- mined at all; one orbit was excluded because of its very high inclination $i$. For these 105 objects, orbits have therefore been derived by more devious means, generally by fixing $e$ (and sometimes $a$ instead or in addition) at reasonable values. The philosophy for this procedure is that, if the residuals from such an orbit are acceptable, the orbit should be closer to the truth than one determined by more direct means that gives values of $e$ and $a$ out side their acceptable ranges. While this is undoubtedly true, it is difficult to judge the reliability of these " $e$-assumed orbits" statistically. Some insight may, nevertheless, be obtained from the following examples of choices between accepting the straightforward class 4 orbits and orbits with assumed eccentricity.

First, for 2223 P-L four different orbits were available:

\begin{tabular}{lccccl}
11 & 11 & $i$ & $i$ & $a$ & \multicolumn{1}{c}{ Computer } \\
\hline 110.46 & 221899 & 1.37 & 0.2747 & 2.0968 & Herget \\
119.18 & 218.75 & 1.66 & 0.2663 & 2.2272 & Bardwell \\
115.50 & 220.07 & 1.52 & 0.2692 & 2.1629 & Marsden $(c$ issumed $)$ \\
112.14 & 221.26 & 1.42 & 0.2730 & 2.1193 & Marsden
\end{tabular}

The orbit computed by Herget, derived according to his method (Herget, 1965) and the quasi-least-squares differential correction described in PLS I, used all six available observations (covering a 4-day arc), and $a$ is just below the acceptable minimum. Bardwell used the same method without the differential correction, and this forces the first and last observations to have exactly zero residuals; on the other hand, he rejected one of the observations and obtained a result that is certainly acceptable. The $e$-assumed orbit incorporated all six observations but was designed to give $a$ in the range expected of normal minor planets. Although certainly not true of all $e$-assumed orbits, a full least-squares differential correction then succeeded in this case and gave a value of $a$ that is just technically in the acceptable range. One concludes that the orbit of $2223 \mathrm{P}-\mathrm{L}$ is basically determinate but that it may be influenced by a rather poor position; the $e$-assumed orbit is undoubtedly a good approximation to the 
truth, although in this case the complete least-squares solution has been adopted.

In the case of $4540 \mathrm{P}-\mathrm{L}$ satisfactory orbits could be derived for eccentricities covering the range $0.00-0.35$, small values of $e$ being associated with large values of $i$ and vice versa. On the other hand, the semimajor axis varied only slightly among the orbits. It is probable that the eccentricity is in fact larger than the value $(0.01)$ eventually adopted, but it is clear that, except for $a$, which must be in the range 3.0-3.2 AU, nothing can really be said about the orbital elements of this object.

For 6339 P-L the eccentricity of the adopted $e$-assumed orbit $(0.24)$ is essentially the smallest that gives acceptable residuals. Larger values, up to $e=0.40$ and more, also represent the observations satisfactorily. It is possible that the omission of one of the observations would lead to a general solution with an acceptable eccentricity, but the orbit of 6339 P-L is obviously not particularly determinate.

The conclusion is that, while in some cases the adopted $e$-assumed orbits may be good approximations to the truth, in many others they are merely wild guesses. There is no way to remedy this situation, but the alternative of giving general results with meaninglessly high eccentricities seems somehow less satisfactory. While the genuine fourth-class orbits can be discussed statistically in a reasonably appropriate way, as is shown in the next section, many of the individual cases must clearly be suspect and in that sense differ from the $e$-assumed cases only because the general solutions just happened to give values of $e$ and $a$ in the acceptable ranges.

\section{COMPARISON WITH THE PLS I CLASS 4 ORBITS}

Although it can be expected that, on the whole, the addition of a second position from a blink pair has increased the accuracy of the fourth-class orbits, it is desirable to put this qualification on a quantitative basis. To do this, the new class 4 orbital elements were compared with those given in PLS I. Since the accuracy of the old orbits had already been discussed, it was hoped that the accuracy of the new orbits could be derived from the differences. For this comparison 370 orbits were available.

It soon turned out, however, that there is a systematic difference between the semimajor axes of the old and the new orbits. Closer inspection showed that this difference is limited to orbits with $a<2.6 \mathrm{AU}$ in PLS I. The average value of this difference is as much as $0.121 \mathrm{AU}$, in the sense that the old values are smaller than the new. Accordingly, for the purpose of determining the accuracy of the new orbits, the comparison was restricted to the cases that originally had $a>2.6 \mathrm{AU}$. No further systematic differences were found, and the following average differences were derived:

$$
\begin{aligned}
|\overline{\Delta a}| & =0.070 \mathrm{AU} \\
|\overline{\Delta e}| & =0.040 \\
|\overline{\Delta i}| & =1.35 .
\end{aligned}
$$

Compared with the corresponding values (derived by comparison of first-class orbits with effective fourth-class orbits determined from the September observations alone $)$ in PLS I $(|\overline{\Delta a}|=0.071 \mathrm{AU},|\overline{\Delta e}|=$ $0.035,|\overline{\Delta i}|=0.76$ ), this gives the impression that the new class 4 orbits are of very good quality. As indicated in the previous section, this is certainly unrealistic. The accuracy of the class 4 orbits in PLS I was probably overestimated, since it was based on measurements of bright images that have smaller positional errors than faint ones. Moreover, the two sets of data are not completely independent.

On the other hand, in 19 cases it was possible eventually to identify further observations that allowed new class 4 orbits to be extended to class 1 . The sample is small, but the resulting differences between the new class 4 and the corresponding new class 1 orbits are:

$$
\begin{aligned}
|\overline{\Delta a}| & =0.040 \mathrm{AU} \\
|\overline{\Delta e}| & =0.027 \\
|\overline{\Delta i}| & =0.70 .
\end{aligned}
$$




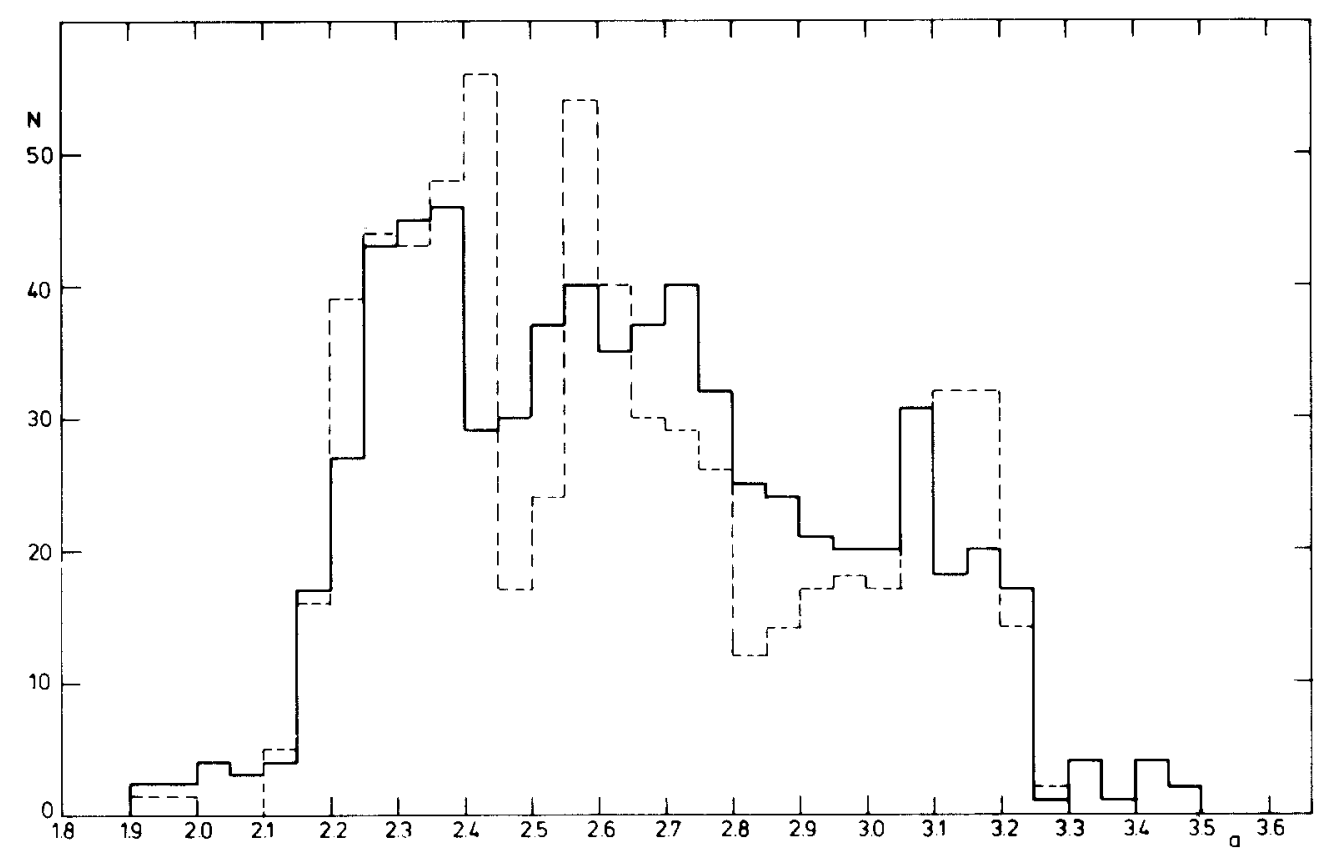

FIG. 1. Firequency distribution of the semimajor axes of the class 4 orbits (solid line) and class 1 orbits (broken line, and scaled to the same number).

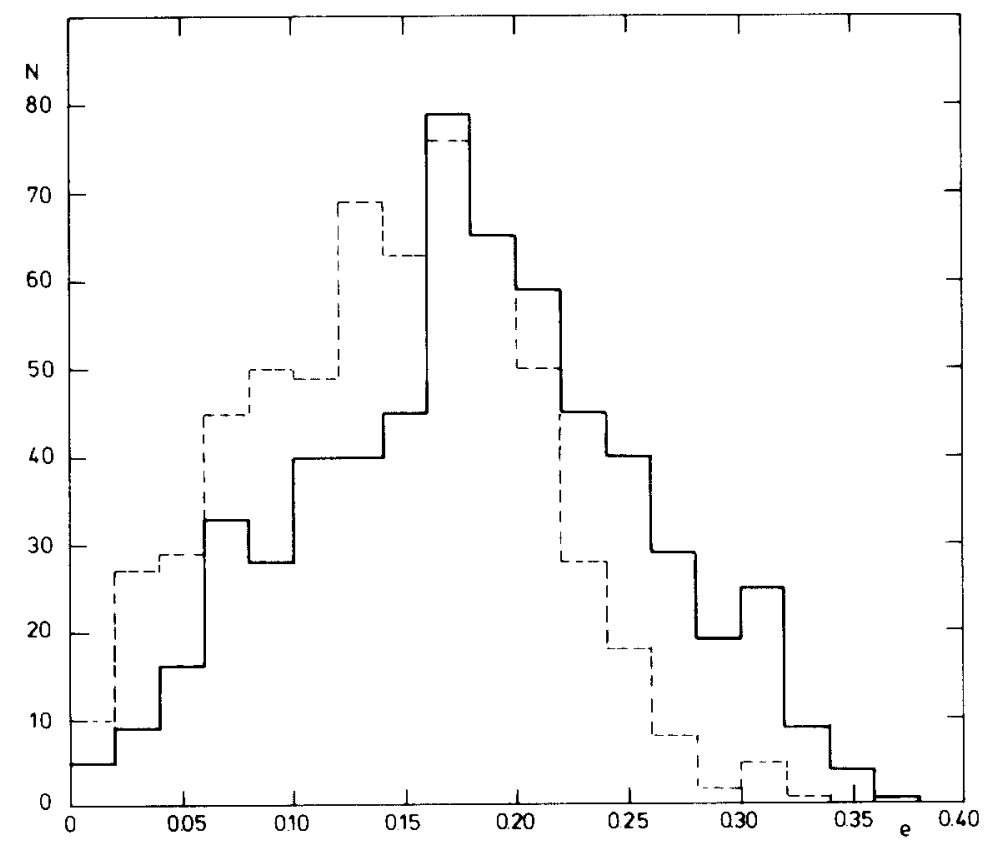

FIG. 2. Frequency distribution of the eccentricities of the class 4 orbits (solid line) and class 1 orbits (broken line. and scaled to the same number). 
DISTRIBUTION FUNCTION OF SEMIMAJOR AXES AND ECCENTRICITIES OF CLASS 4 ORBITS

The Figs. 1 and 2 show the distributions of the semimajor axes and eccentricities of the final PLS II orbits of classes 1 and 4, the results of class 1 being shown with a broken line and those of class 4 with a solid line. Only representative samples of the class 4 orbits have been plotted (681 in the case of the semimajor axes and 590 for the eccentricities), and the class 1 entries have been scaled to correspond to the selected number of class 4 orbits.

In the plot of semimajor axes (Fig. 1), the $3: 1$ Kirkwood gap is clearly visible (around $2.5 \mathrm{AU})$, although in the class 4 distribution it is much more shallow than in that of class 1. The distribution function for the eccentricities (Fig. 2) is rather similar for the two quality classes, each having a maximum near $e=0.17$.

\section{ORBITS OF QUALITY CLASS 2}

A class 2 orbit, which involves observations over an arc of from 4 to 9 days in one of the observing months and just a single observation in the other, is in effect a case where it is not clear whether the orbit has essentially the accuracy of a class 1 orbit or whether it should really be treated as of only class 3 or 4 . Even if the residuals are satisfactorily small, one can never be absolutely sure that the observation and/or its identification are correct. Statistically, the distribution of semimajor axes is found to be more nearly similar to that of the class 1 orbits than to that of class 4 . In particular, the $3: 1$ Kirkwood gap is essentially in its right place. It can thus tentatively be concluded that the additional position generally really does belong to the object to which it has been assigned. In fact, the number of class 2 orbits in PLS II is less than in PLS I, for it was possible to promote almost 50 of the earlier orbits to class 1 .

On the other hand, the large residuals shown in PLS I indicate that the month-to- month linkages of observations of 2202 , 2207, 4864, and 6692 P-L were incorrect. Further investigation has also revealed incorrect linkages for 2785 and 6794 P-L (the latter in fact originally was a class 1 orbit). Accordingly, these orbits have now been relegated to class 4 status.

\section{SPECIAL ORBITS}

Orbits for nine new Trojans (2804, 4292, $4322,4534,6375,6889,9602,9612$, and 9616 P-L) are listed in this paper. Four of these were suspected to be Trojans in PLS I, but it was not then possible to give Trojan-like orbits. Unfortunately, all but one of the new Trojans have $e$-assumed orbits. While this is regrettable, it does provide a good illustration of the occasional great usefulness of this method of orbit computation. One more Trojan, 2706 P-L, which had a class 4 orbit in PLS I, now has a class 1 orbit.

Three new Hilda-type objects (2864, 6240 , and 7617 P-L) were found, two of them again with $e$-assumed orbits (7617 P-L had a rejected orbit in PLS I). Three earlier class 4 Hilda orbits $(2709,4710$, and 6847 P-L) have been somewhat improved, but they remain class 4 .

Four new Hungarias (3006, 3509, 6310, and 6378 P-L) were found (two again with $e$-assumed orbits), as was the known Hungaria object $3566 \mathrm{P}-\mathrm{L}=$ (1235) Schorria. Two of the earlier class 4 Hungaria orbits (2112 and 4761 P-L) have been somewhat improved, and three more (7071, 7072 , and 7082 P-L, the first of which has recently been identified at other oppositions and numbered) have been improved to class 1 quality.

The orbit of one more fast-moving object, 5025 P-L, was obtained. At the time of observation this object was evidently some 0.7 AU from Earth. If the elements are reliable, this object would have its perihelion in the vicinity of the orbit of Mercury and its aphelion between the orbits of Jupiter and Saturn! 


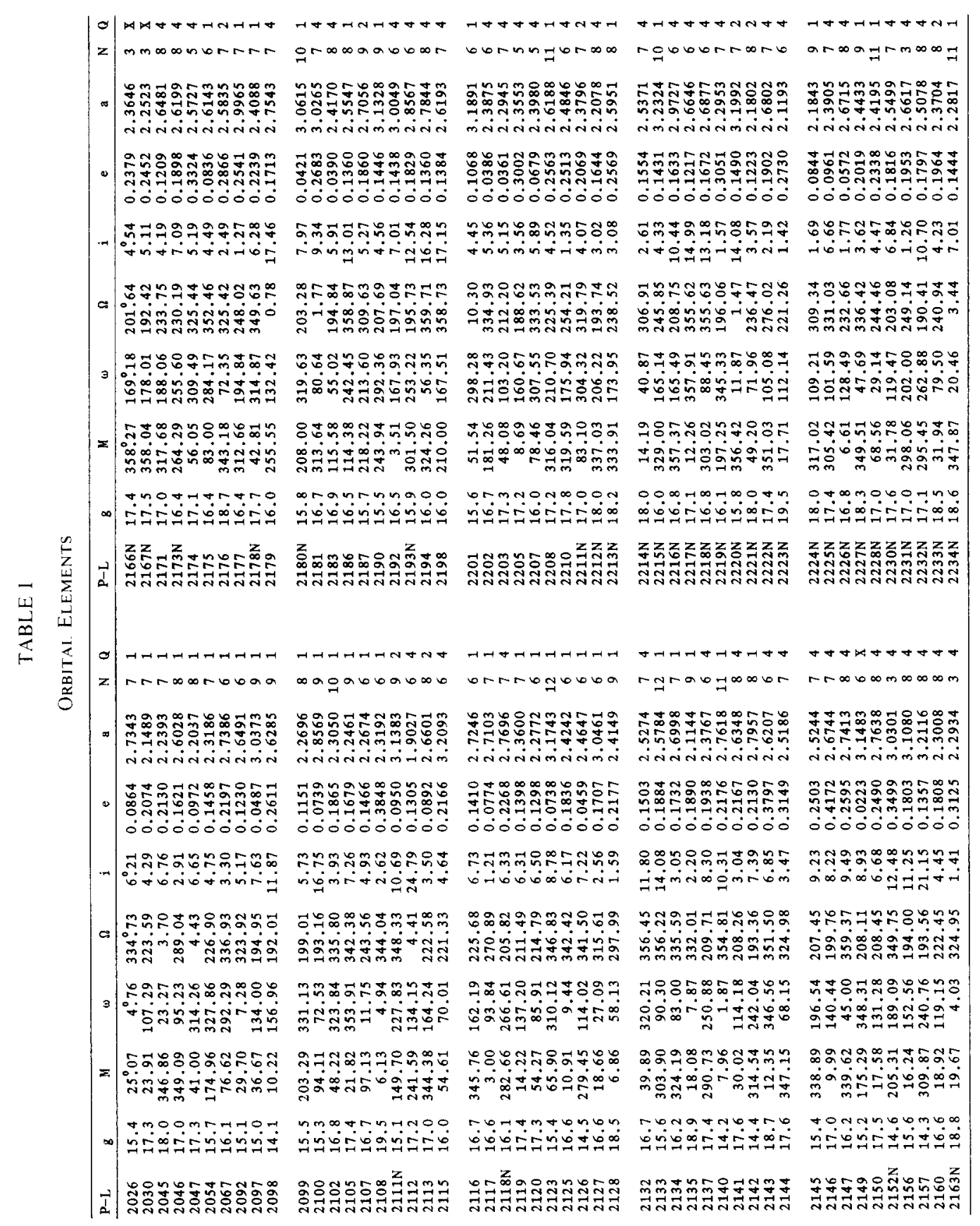




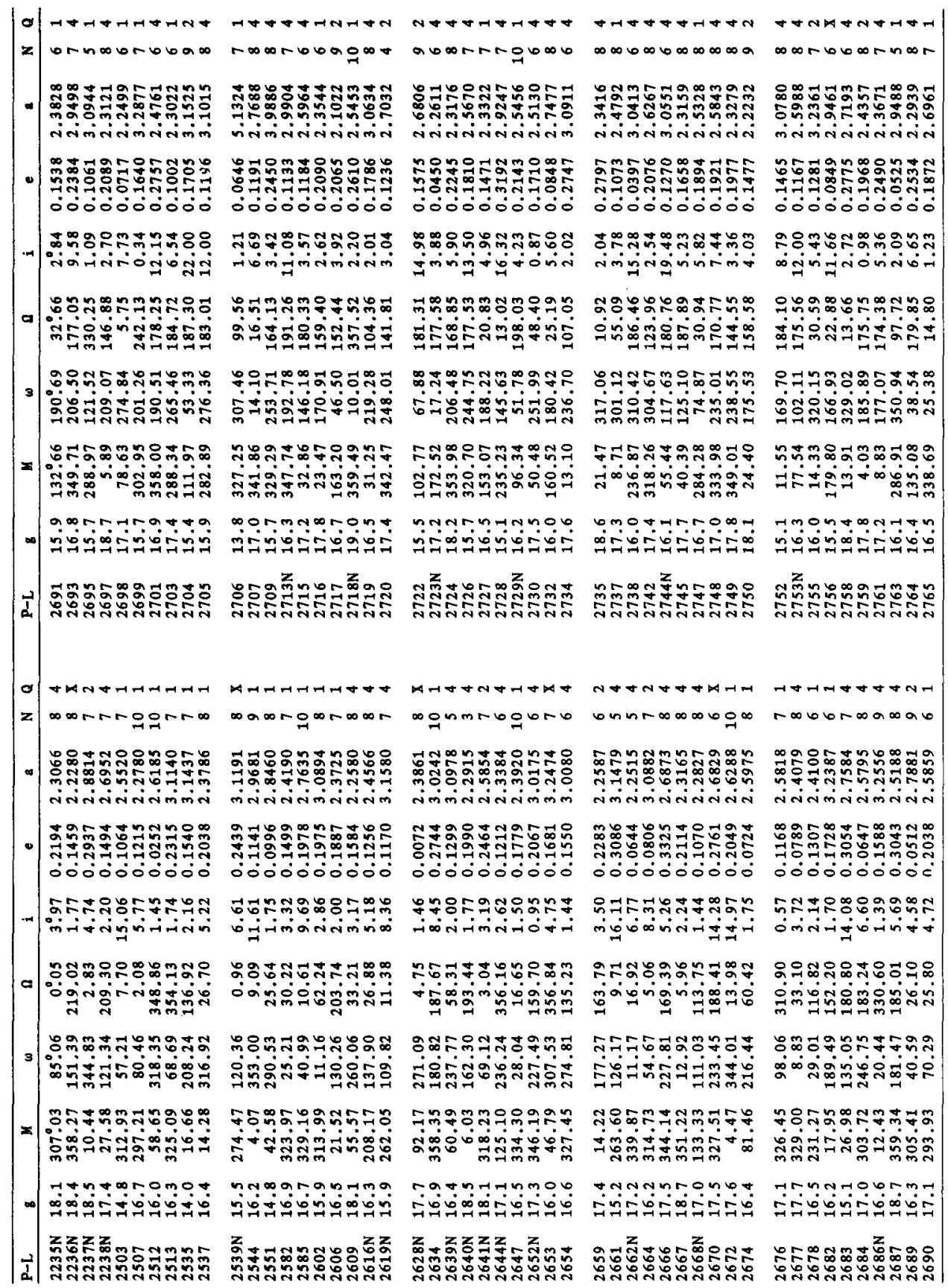




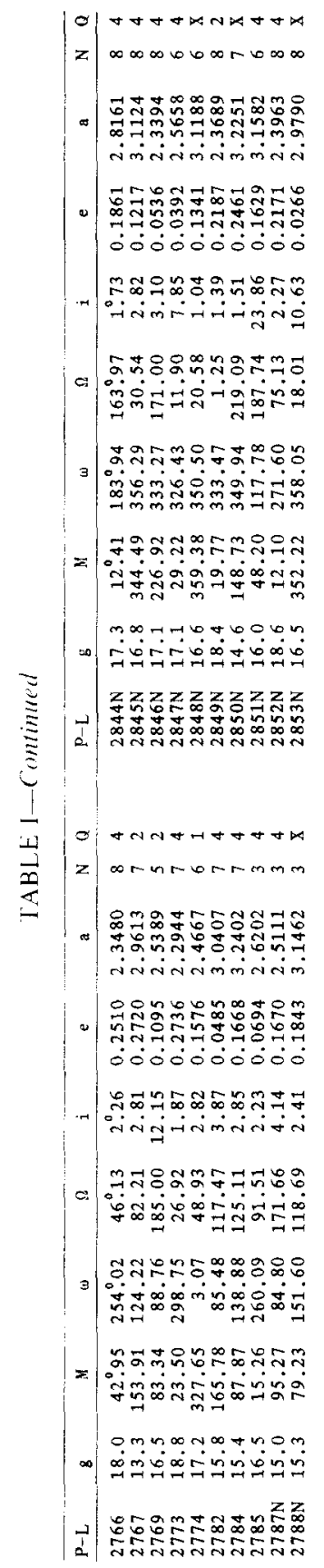

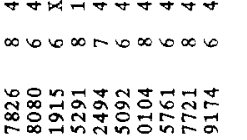
inimiriminim

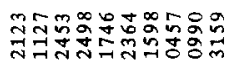
000000000.

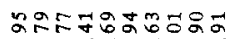
نboviciosonit

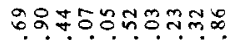

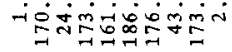

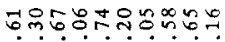

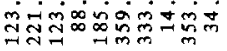

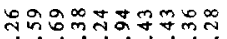

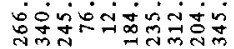

Nootunatmor.

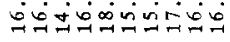

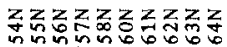
Nin

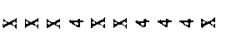
mmenmmento

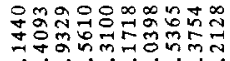
miniviminivin

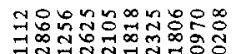
0000000000

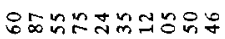

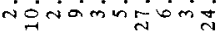

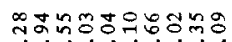

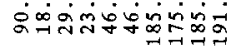

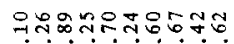

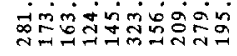

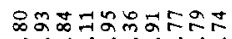

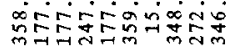

O.०manm?tm.

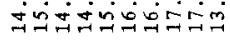
zzzzzzㅜㅇㅢ

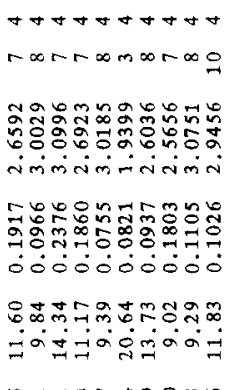

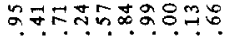

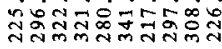

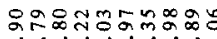

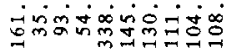

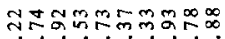

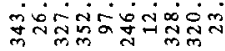

o OObHnOOn

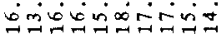

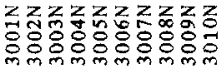

tantatathat $\infty=000460 \infty$

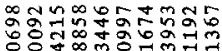
miniरivininimo

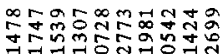
0000000000

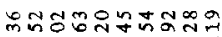

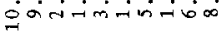

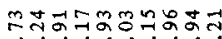

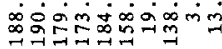

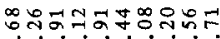
:

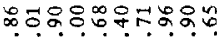

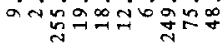

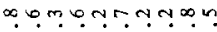

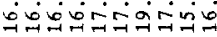

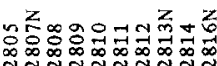

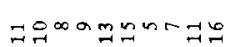

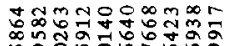
iniminisinin

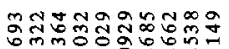

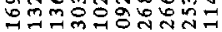
00:000ं0்:

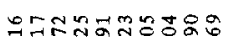

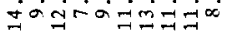

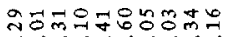

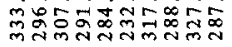

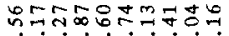

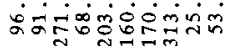

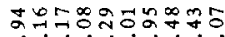

-

$\forall a n+a O N \infty \Rightarrow a$

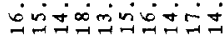

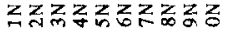

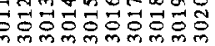

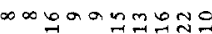

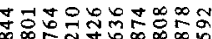

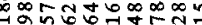
miniviminism

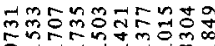
000000000.

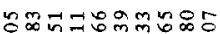

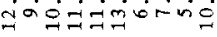

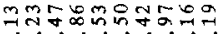

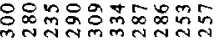

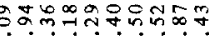

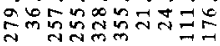

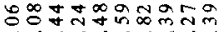

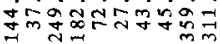

tann $0000 \mathrm{nt}$ Inviduniving

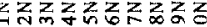

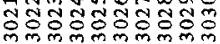

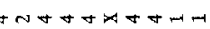
otrinosana

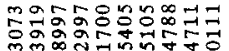

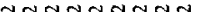

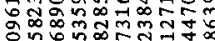

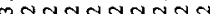

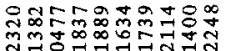
0000000000

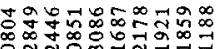
年

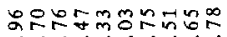

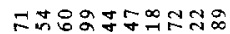

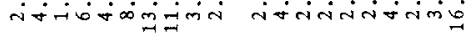

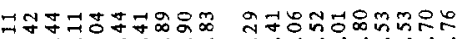

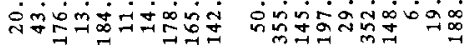

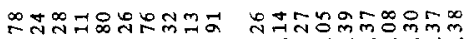
mơno

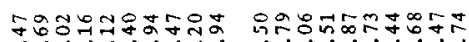

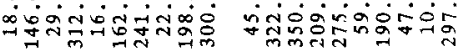

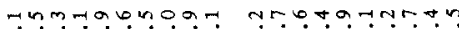

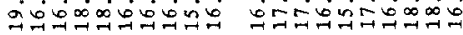
roa

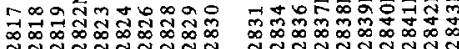




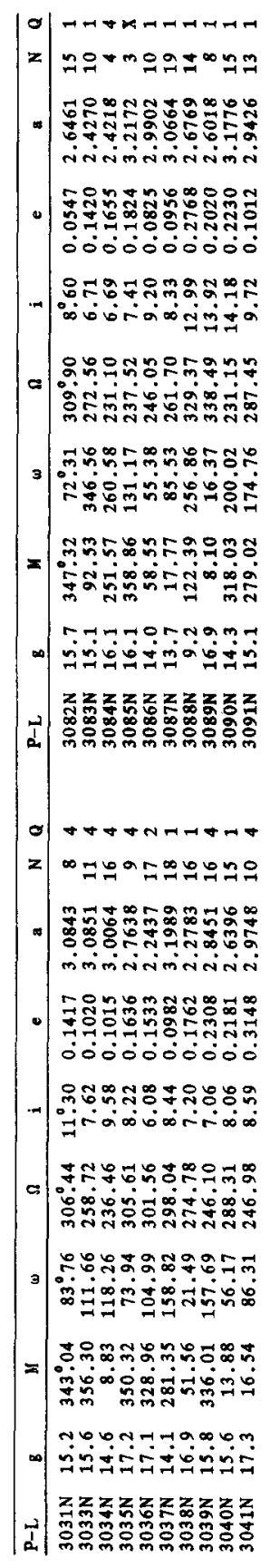

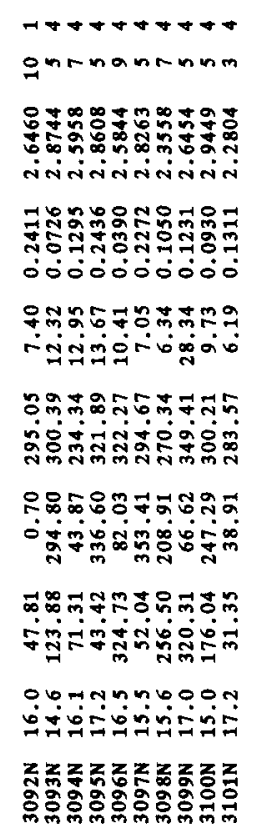

h4t-4attath

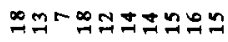

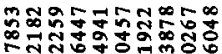
Niviniminimin

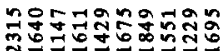
0000000000

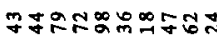
ooringigosa

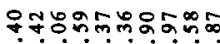

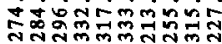

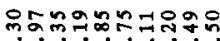

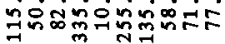

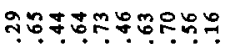

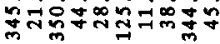

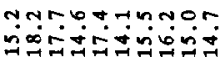

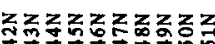

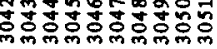

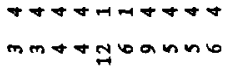

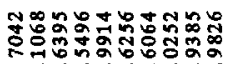

Nininininimiñ

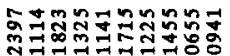
00000.000.0

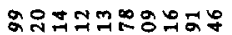

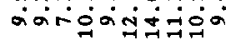

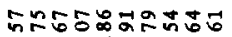

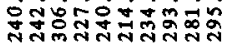

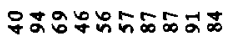

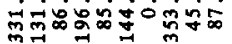

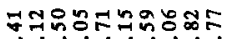

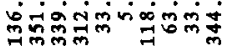

ntartoonalo

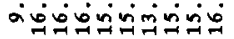

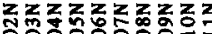

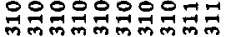

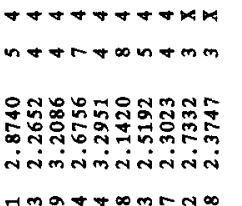

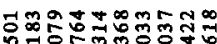

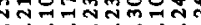
0000000000

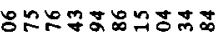

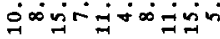

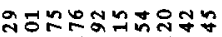

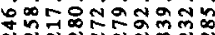

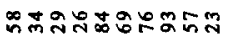

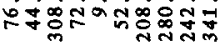

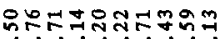

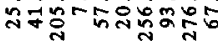

$m \rightarrow \infty-6 n 0 a 0 m$

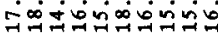

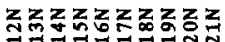

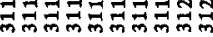

국퓨

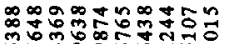

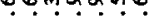
तininininiminis

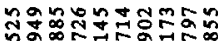

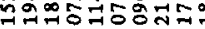
0000000000

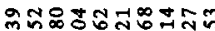

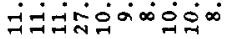

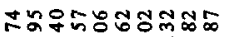

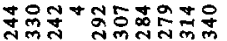

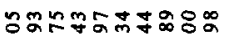

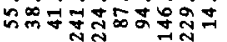

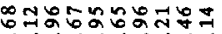
तु

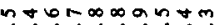

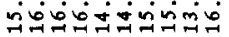

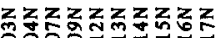

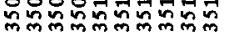

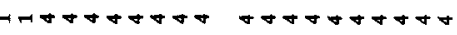

nzarmotar

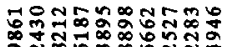
तinininininin

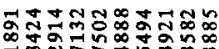
iminivininiñ

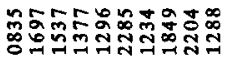
0000000000

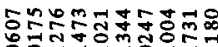
000000000

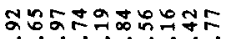

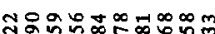
vibovisuninivion ำㅇํㅇ요요용

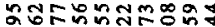

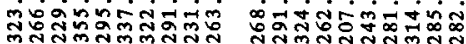

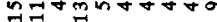

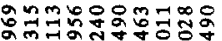
तiniminininim

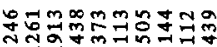
000000000

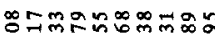

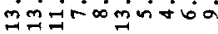

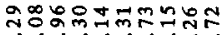

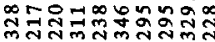

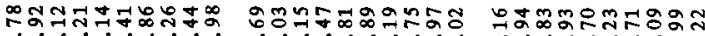

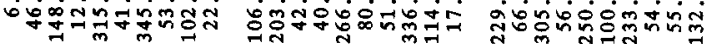

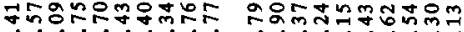
iming

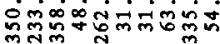

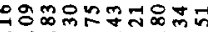
gioninimint

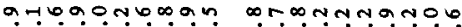

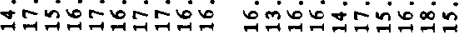

mingo:

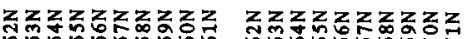

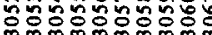

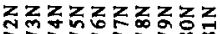
: 


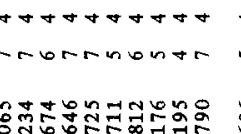

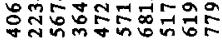
inivivinivivin

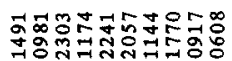
00000000000

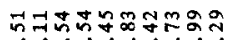

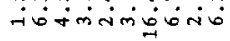

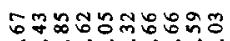

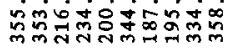

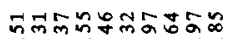

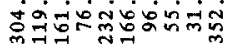

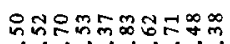

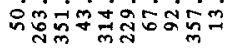
-woantarar

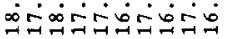
중주의

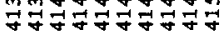

nromenothr
nd

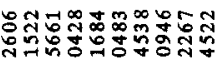
iniviniminimi

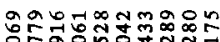

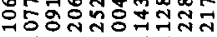
0000000000

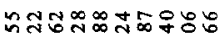

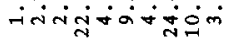

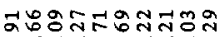

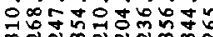

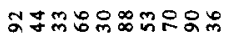

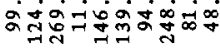

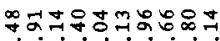

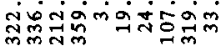

$\ln \infty$ onn $\ln \pi 0$

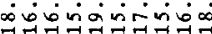

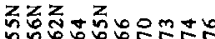

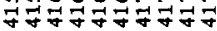

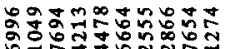
iminicicicicis

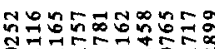

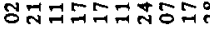
0000000000

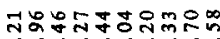

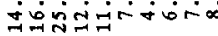

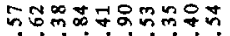
సี่స

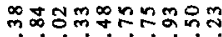

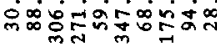

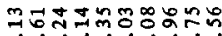

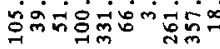

$960007900 m$

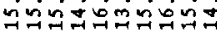

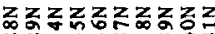

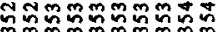

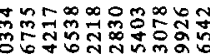
minivininive

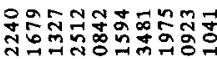
1000000000

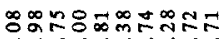
में nn:

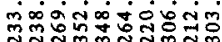

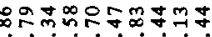

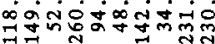

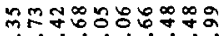

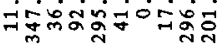
Nunogonong

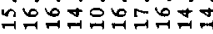

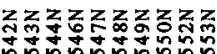

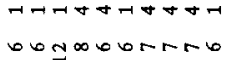

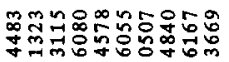
iminiriniñin

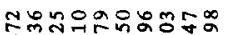

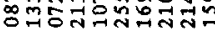
0000000000

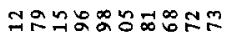

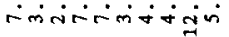

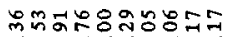

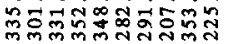

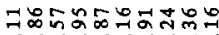

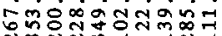

马눙

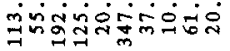

$m \infty \forall 0 \infty 00$ or 0 -

웅-

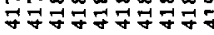

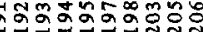

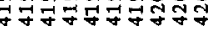

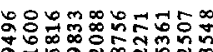
जलितंNinimiñ 000000000

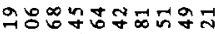

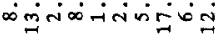

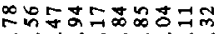

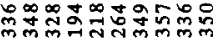

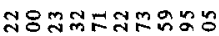
ल্ল

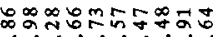
-

ominonmation

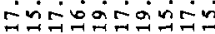

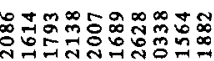

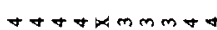

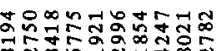
तininimimitie

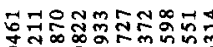
0000 00000

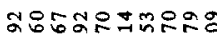
nंt $+\infty+\infty \sin ^{\infty}$

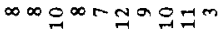

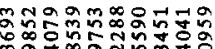
तinininividim

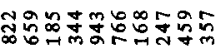

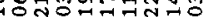
0000000000

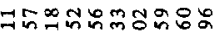

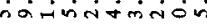

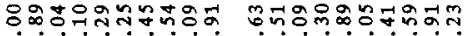

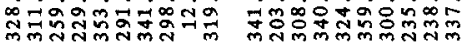

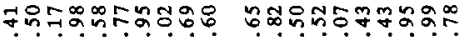

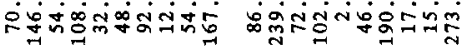

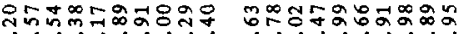

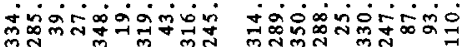

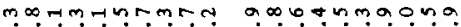

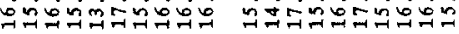

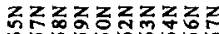

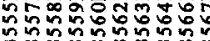




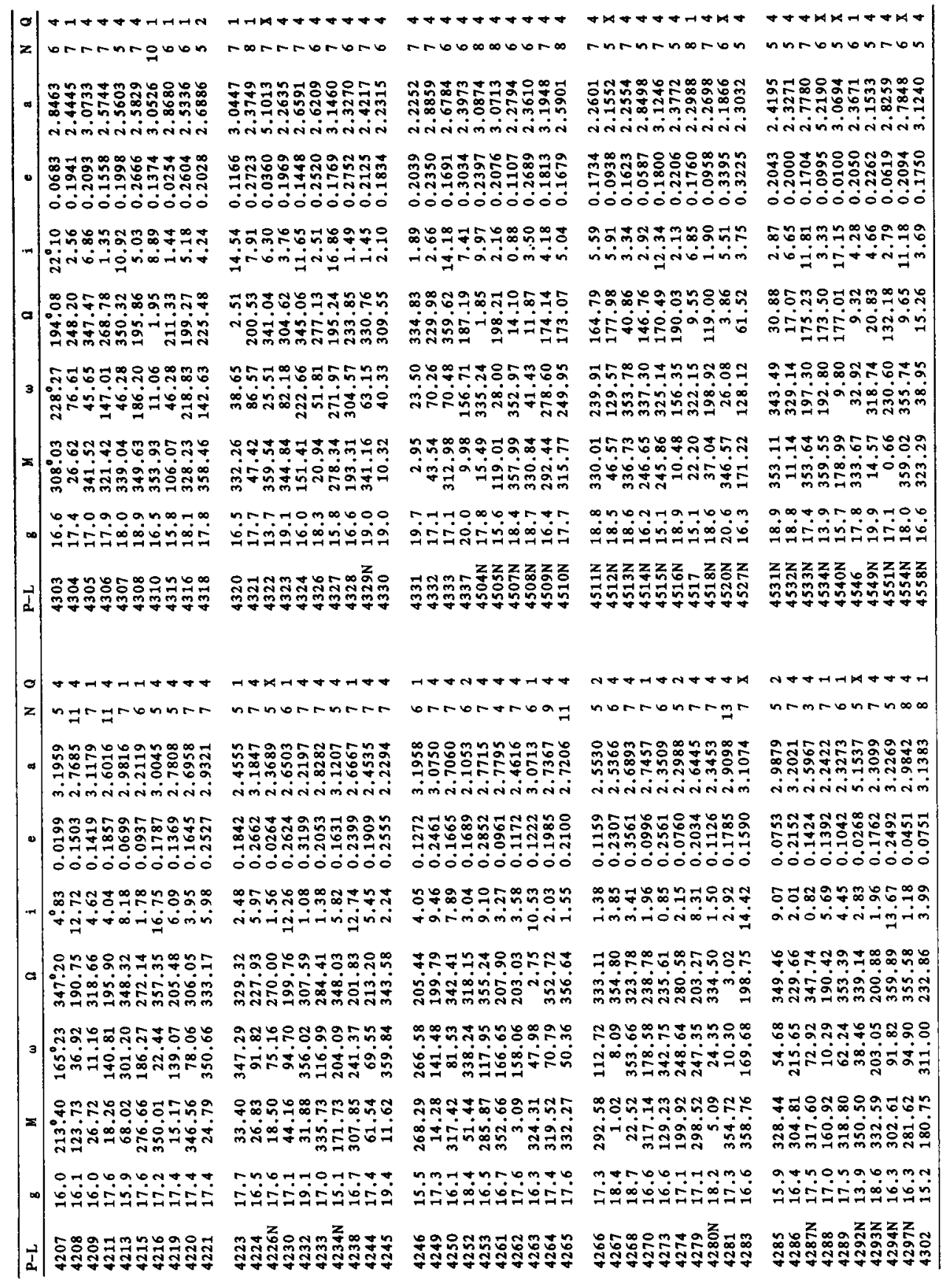




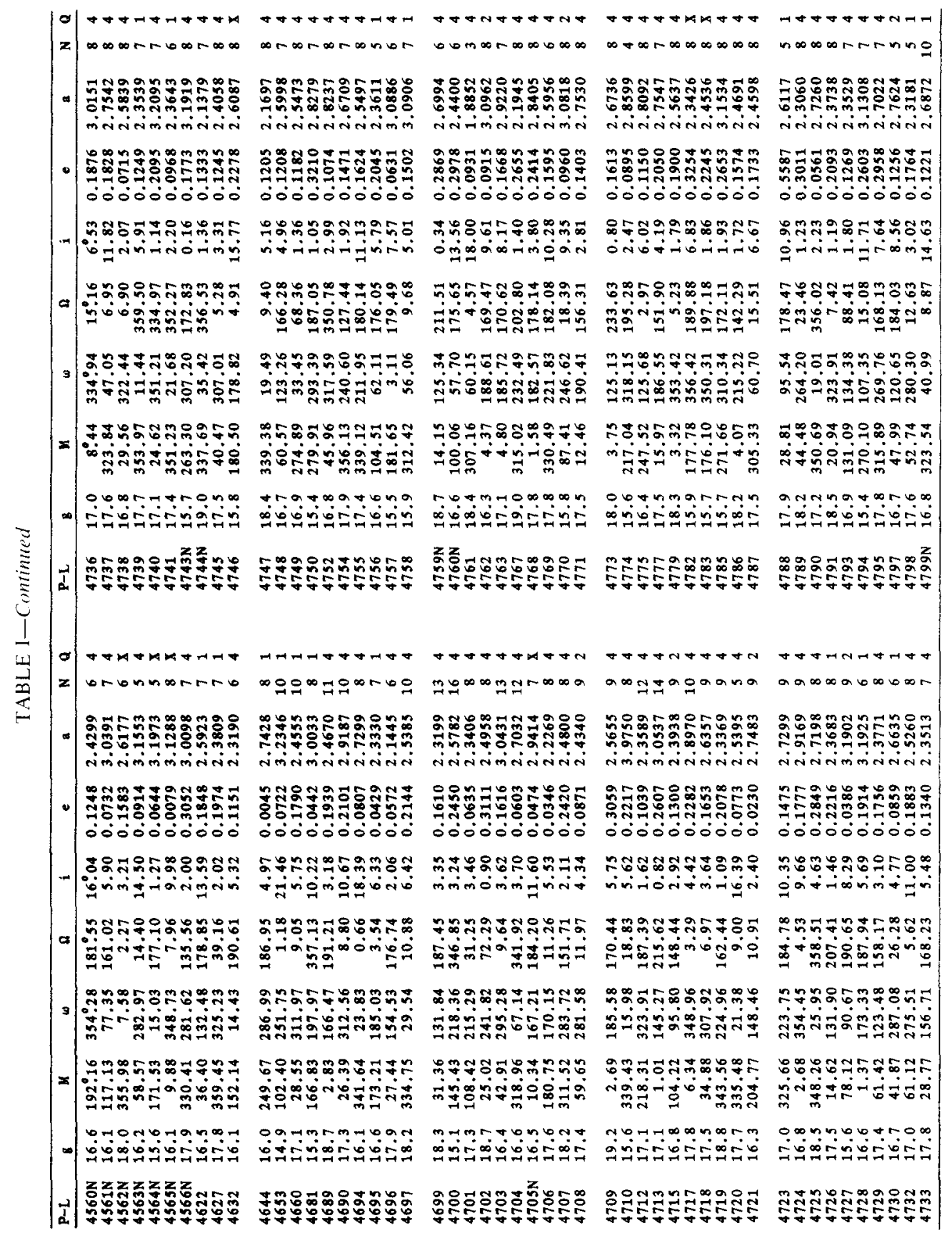




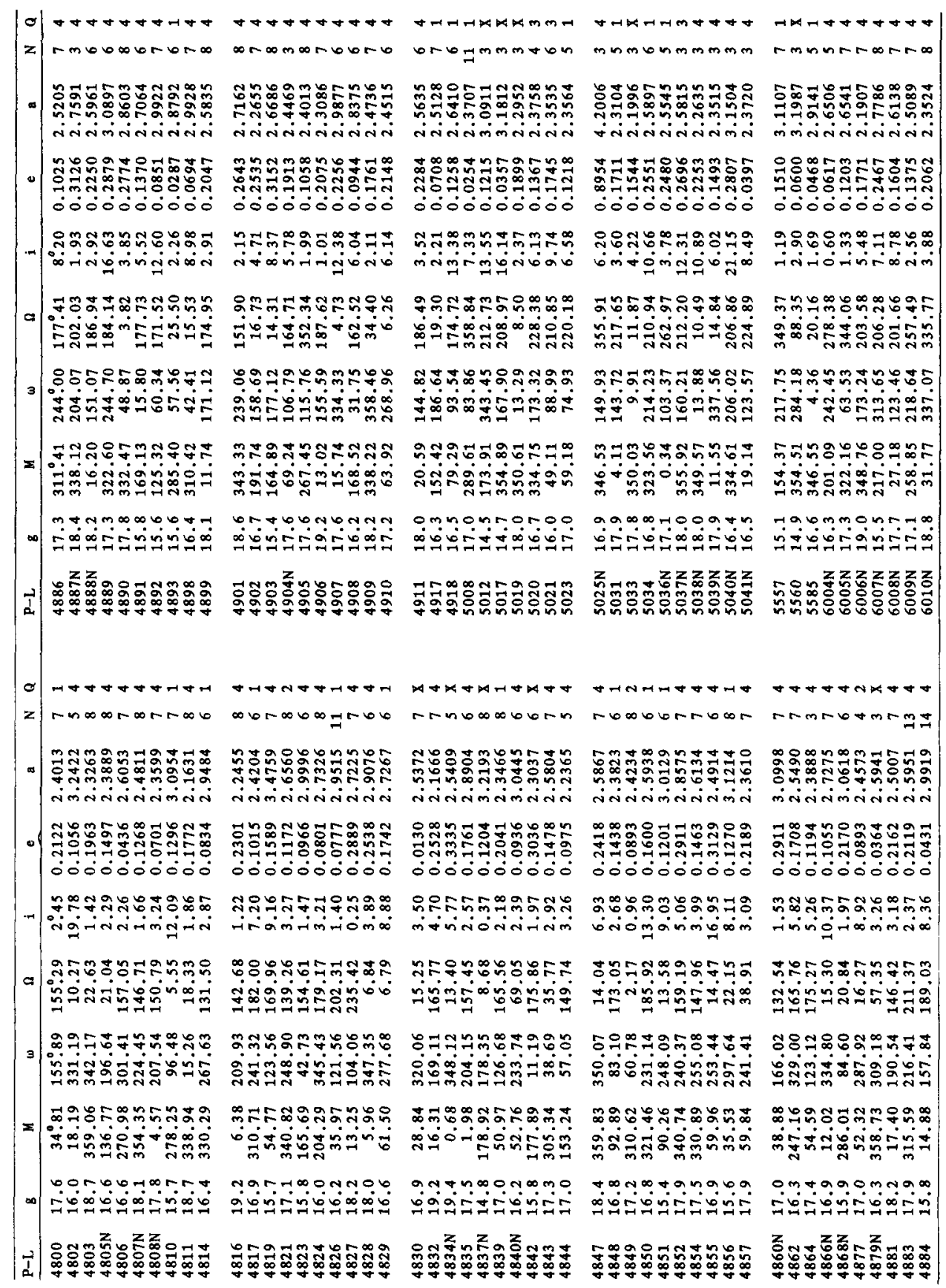




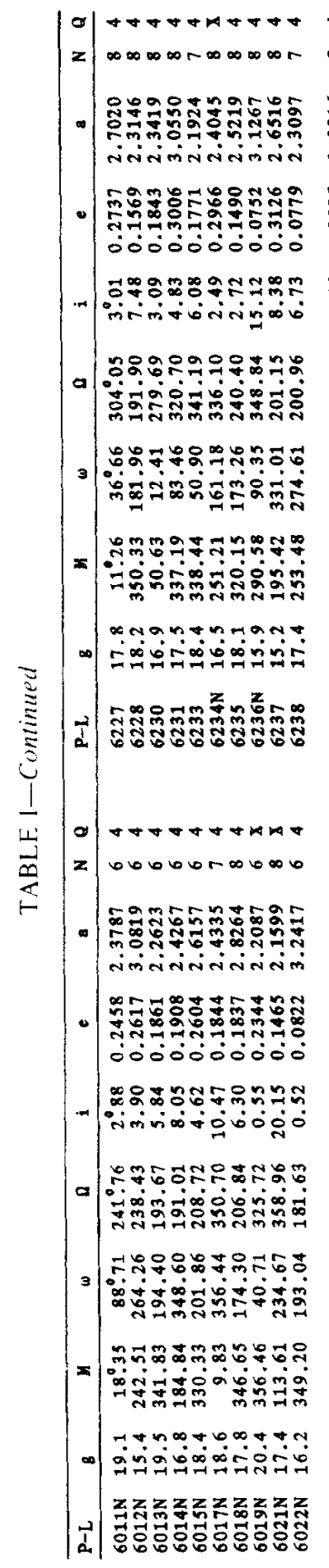

$\Delta \pi t-\pi, t+t+t$

$\infty \infty+\infty \infty+\infty \infty \infty$

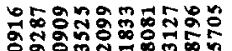

miniminiminiñ

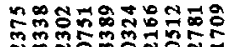

00000000000

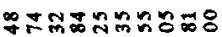

roosirivivis

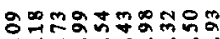

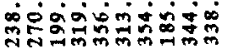

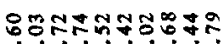

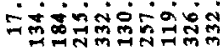

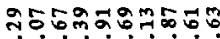

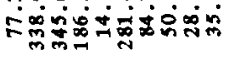

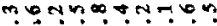

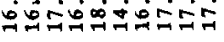

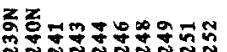

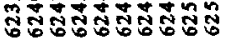

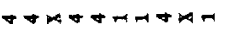

r-

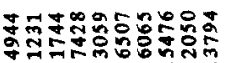
iminininine

mo: 0000000000

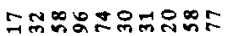

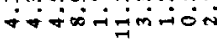

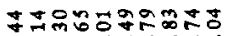

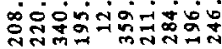

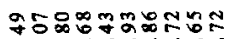

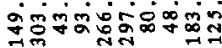

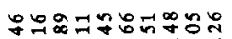

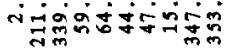

$+0,0 \infty-100,0$

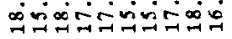
준증중 ํํㅇํㅇㅇํㅇㅇㅇㅇํㅇㅇำำำ

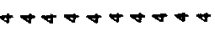

- $000000000 \mathrm{NL}$

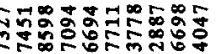
ininicininivin

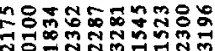
กิธก 000000000

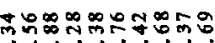
simmininimini

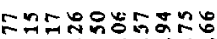

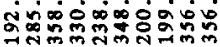

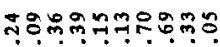

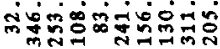

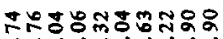

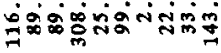

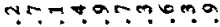

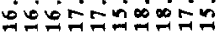
n் กู่

$\infty \infty \infty \infty \infty \infty \infty+\infty$

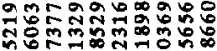
Niniñimininin

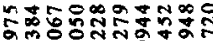

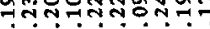
0000000000

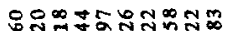
-ibivg-into-

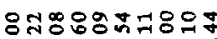

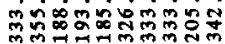

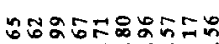

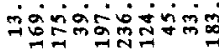

옳워ำำㄷำ

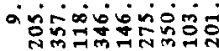

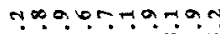

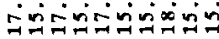

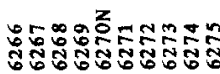
$\infty 0_{\infty} \infty \infty \infty \infty \infty \infty$

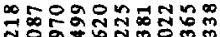

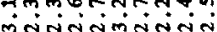

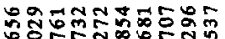

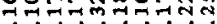
0000005000

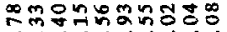

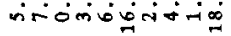

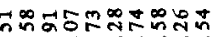

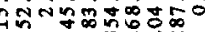

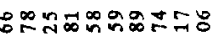

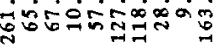

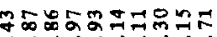

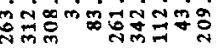
am-r+custa

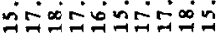

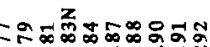

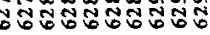

$\min \infty \infty \operatorname{los}_{\infty} \infty \infty \infty$

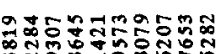
ininiminininin

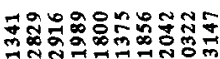
0.000000000

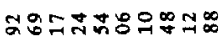
-imitridina

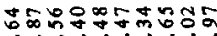

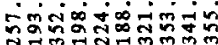

$\infty \infty+\infty \infty \infty \infty \infty \infty$

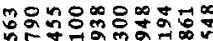

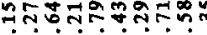
जिiciciniv

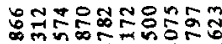
000000050

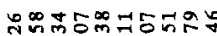

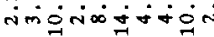

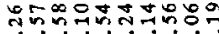

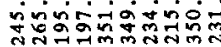

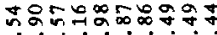

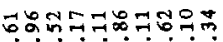

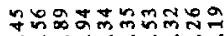

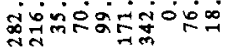

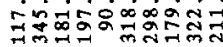

กำ

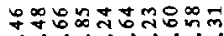

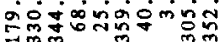

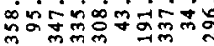

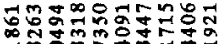

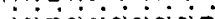

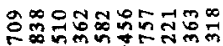

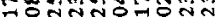
0000000000

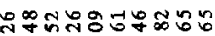

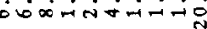

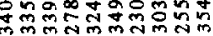

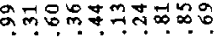

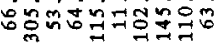

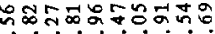

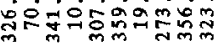
000000000

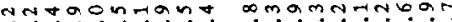

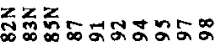

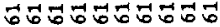

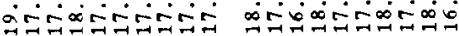

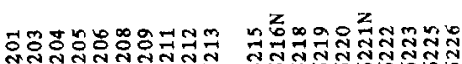




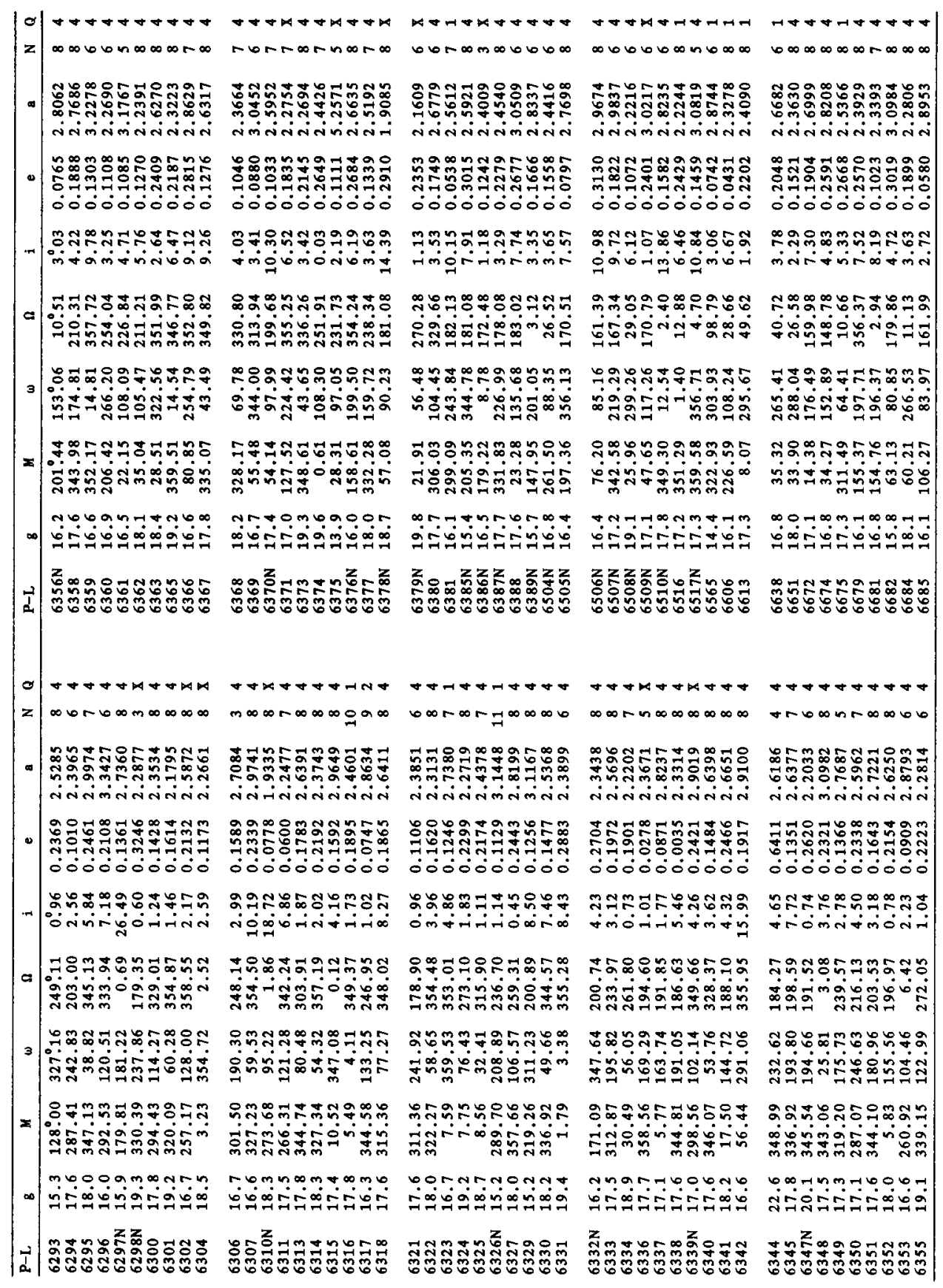




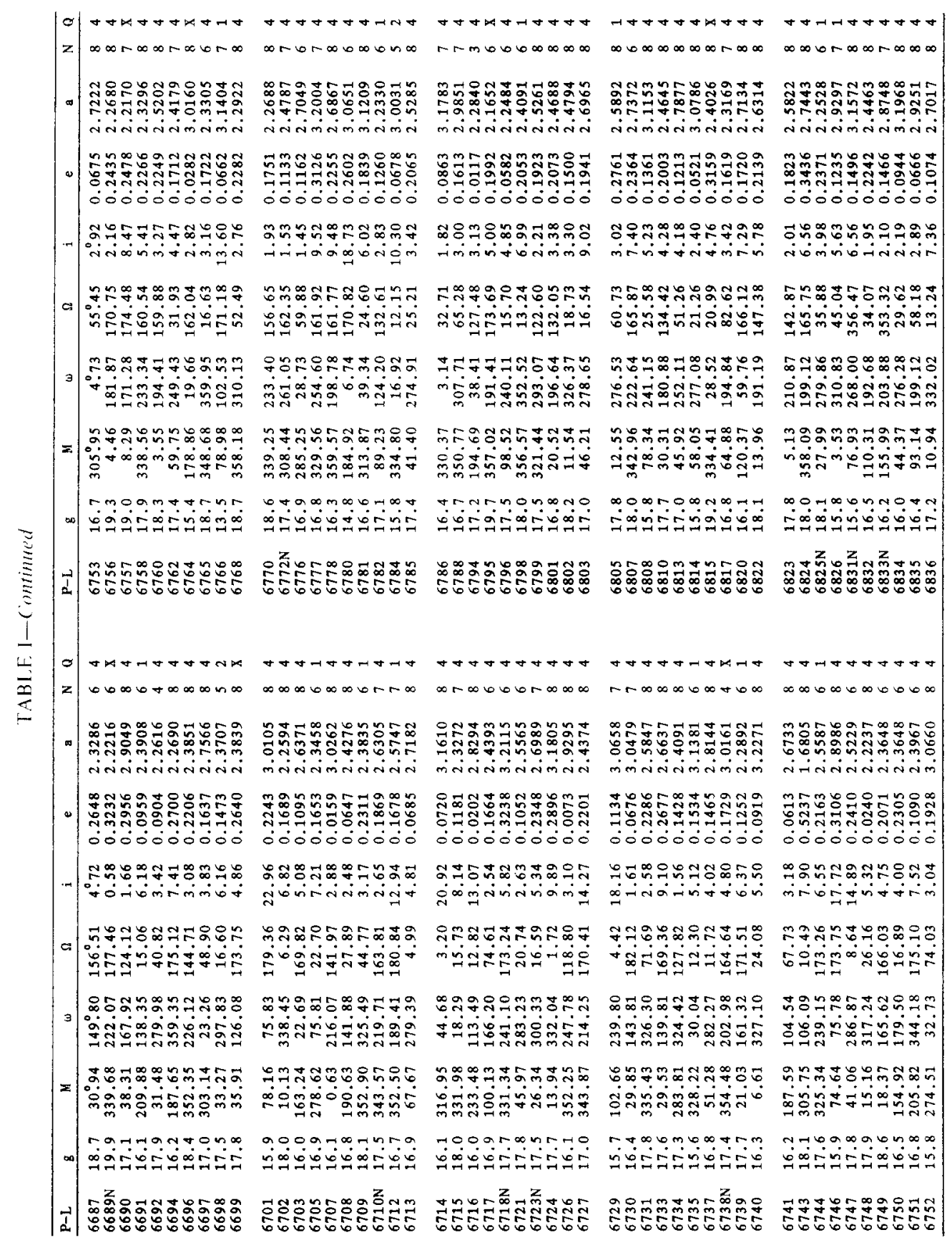




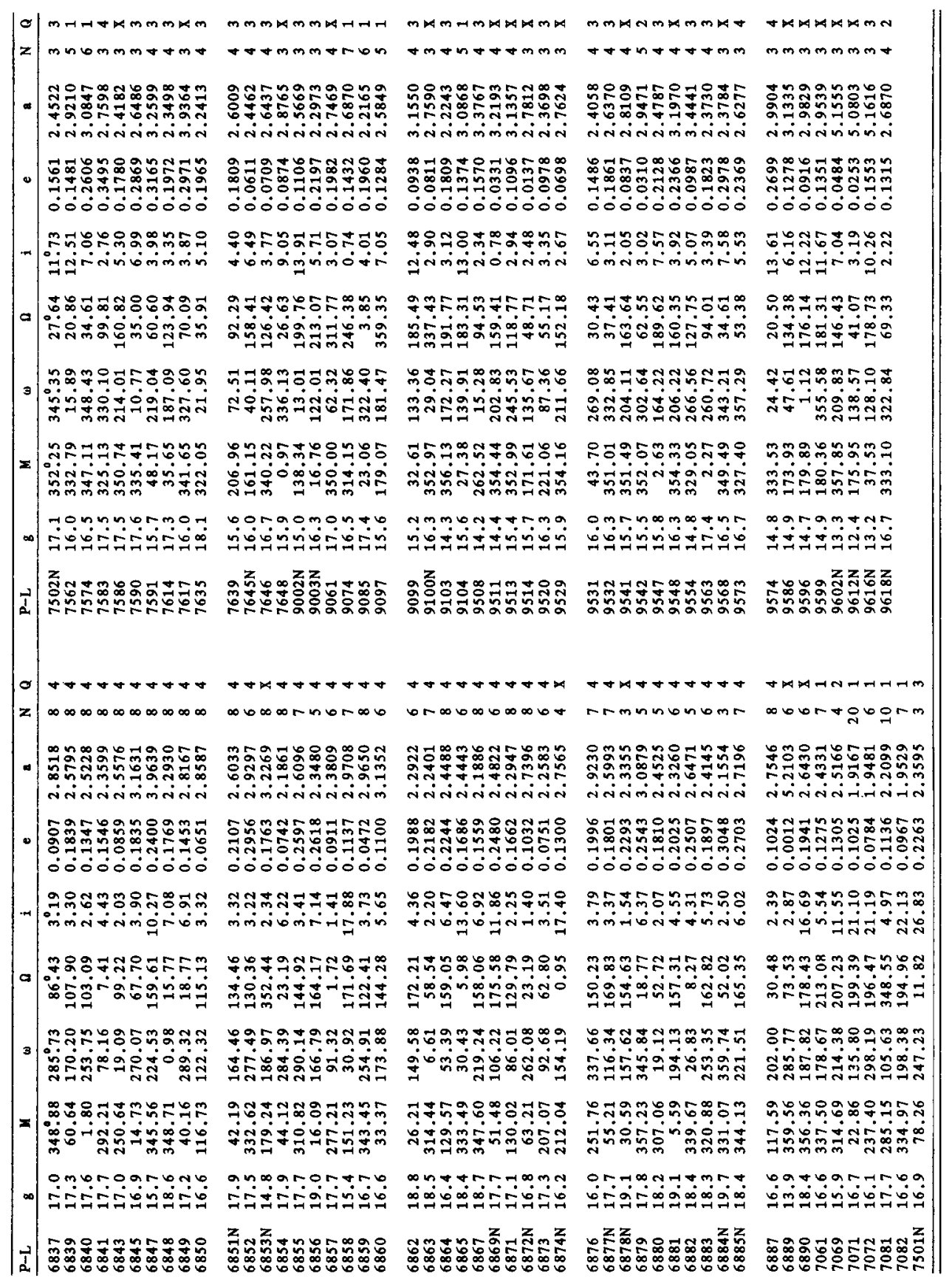


New computations confirm the general correctness of the two previously known Apollo-type objects, 6344 and 6743 P-L (both still of class 4). A class 2 Amor-type orbit in PLS I, that of 4788 P-L, has now been promoted to class 1 , and at $e=0.56$, this is by far the most eccentric class 1 orbit in PLS II.

In a study of orbits in the $2: 1$ Kirkwood gap, Franklin et al. (1975) concluded that most, if not all, of the 21 PLS I orbits (all then of class 3 or 4 ) that appeared to be librating were spurious. This is borne out in PLS II, which in ten instances shows "former librators" with improved general orbits that are far from the gap. In three cases $(2691,2834$, and 5557 P-L) these improved orbits are of class 1 . On the other hand, 2699 P-L has a class 1 orbit of only moderate eccentricity right in the gap. The class 3 orbit for $7591 \mathrm{P}-\mathrm{L}$ also remains in the gap.

The highest orbital inclination among the class 1 orbits is still the 26.4 (for $2104 \mathrm{P}-\mathrm{L}$ ) mentioned in PLS I. Two third-class (the Hungaria 3509 P-L and the Phocaea 7501 P-L) and four fourth-class orbits have higher inclinations, the largest value being 30.7 in the case of $3055 \mathrm{P}-\mathrm{L}$. Since the SA 68 field is further from the ecliptic than the older fields, it is to be expected that it would produce a greater proportion of highinclination orbits. The median inclination of the SA 68 objects is 9.2 , just twice the median inclination for the Survey as a whole.

No proper elements have been obtained for the new orbits, so little can be said about the occurrence of family members among the new class 1 orbits.

\section{TABULATION}

PLS II contains a total of 2403 orbits, and the number of the orbits in each quality class $Q$ is as follows:

\begin{tabular}{lr}
$Q$ & No. \\
\hline 1 & 1124 \\
2 & 132 \\
3 & 183 \\
4 & 859 \\
$X$ & 105
\end{tabular}

Quality class $X$ refers to the $e$-assumed orbits, 96 of which might otherwise be ascribed to class 4 , the remainder to class 3 . It should be noted that $2510 \mathrm{P}-\mathrm{L}=(1694)$ and 6549 P-L $=(1630)$ were accidentally listed under both designations in PLS I. 5029 P-L should also be eliminated, because it is identical with 2128 P-L, which now therefore has a class 1 orbit. The orbit of 6303 P-L is erroneously given as class 4 , instead of class 1 , in PLS 1.

Table I lists 1198 orbital elements that supplement or amend the orbits in PLS I. The standard angular elements $\omega, \Omega$, and $i$ are referred to the mean equinox of 1950.0, and the mean anomaly $M$ corresponds to the epoch JED 2437200.5 = 23.0 Sept 1960 ET. The absolute magnitudes $g$ were calculated using the same phase function adopted for PLS I. $1.03 T(\alpha)+0.039|\alpha|-$ 0.05 , where $T(\alpha)$ represents the opposition effect (as a function of the phase angle $\alpha$ ) tabulated by Gehrels (1967). They are thus not exactly equivalent to standard $B(1,0)$ values. The column $N$ shows the number of observations utilized in the computation. The 441 orbits for which there were no corresponding entries in PLS I are indicated with a letter $\mathrm{N}$ after the planet number.

Thirteen of the objects for which orbits are given in Table I have been identified with long-numbered minor planets or with minor planets observed at other oppositions (some of which have therefore recently been permanently numbered). Better orbits for these objects are obviously available elsewhere. These P-L objects with identifications (numbers in parentheses denoting numbered minor planets) are as follows:

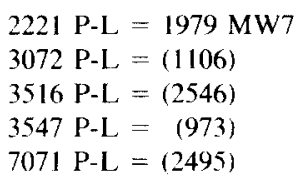

\author{
$3071 \mathrm{P}-\mathrm{L}=(2799)$ \\ $3102 \mathrm{P}-\mathrm{L}=(156)$ \\ $3537 \mathrm{P}-\mathrm{L} .=(2412)$ \\ $4805 \mathrm{P}-\mathrm{L}=1981 \mathrm{EP} 22$
}


Likewise, many of the objects for which orbits were published only in PLS I have subsequently been permanently numbered or identified at other oppositions. These are:

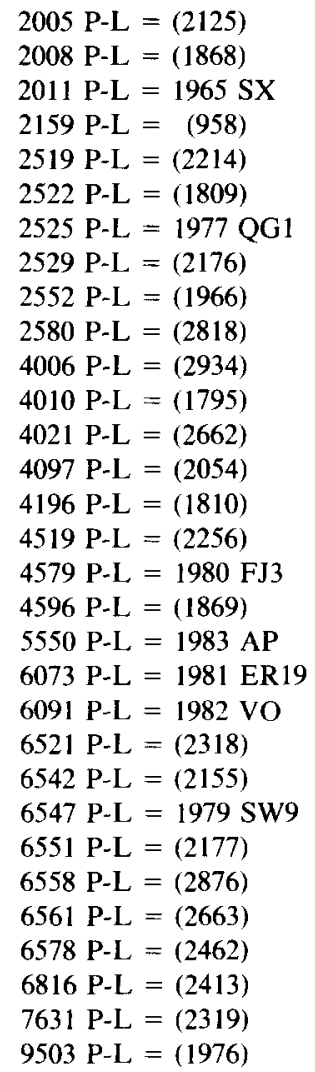

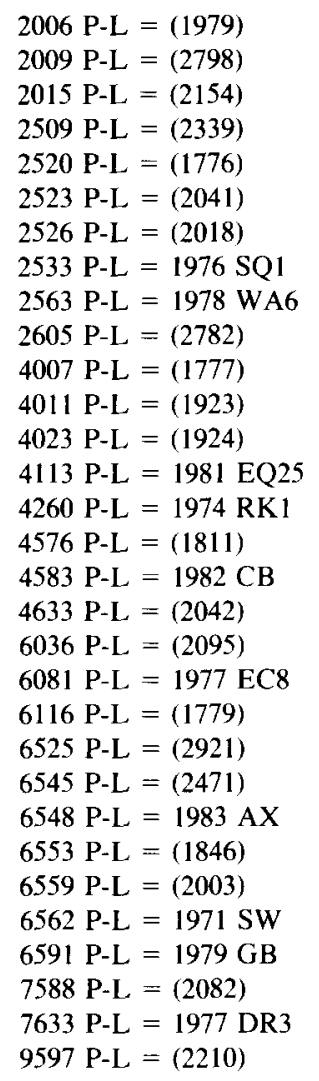

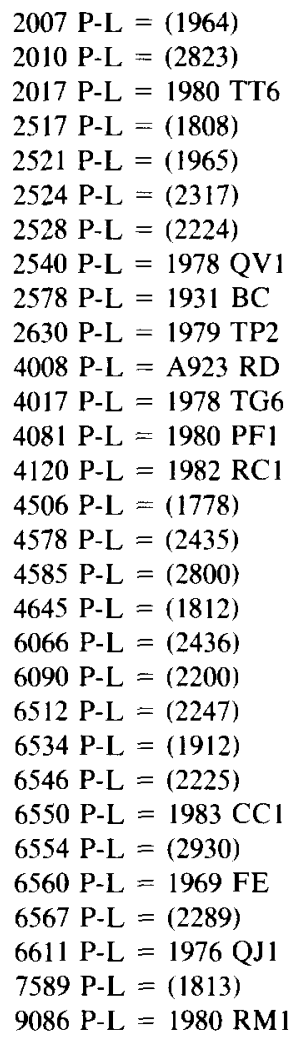

\section{ACKNOWLEDGMENTS}

The authors wish to thank the director of the Lunar and Planetary Laboratory, Tucson, for his willingness to loan the blink microscope to the Leiden Observatory for use in this program, and the director of the Kapteyn Laboratory, Groningen, for making available a plate-measuring instrument. The important contributions of Tom Gehrels (who obtained all the Survey plates with the 1.2-m Schmidt telescope at Palomar in 1960) and of Ingrid van Houten-Groeneveld are also very gratefully acknowledged. Further thanks are due to Conrad Bardwell for the computation of some of the orbits; he has also been responsible for the majority of the identifications of Survey objects at other oppositions.

\section{REFERENCES}

Franklin, F. A., B. G. Marsden, J. G. Williams, AND C. M. BARDWEll (1975). Minor planets and comets in libration about the $2: 1$ resonance with Jupiter. Astron. J. 80, 729-746.

Gehrels, T. (1967). Minor planets: II. Photographic magnitudes. Astron. J. 72, 1288-1291.

Herget, P. (1965). The computation of preliminary orbits. Astron. J. 70, 1-3.

van Houten, C. J., I. van Houten-Groeneveld, P. Herget, and T. Gehrels (1970). The PalomarLeiden Survey of faint minor planets. Astron. Astrophys. Suppl. Ser. 2, 339-448. 